\title{
Formación permanente del profesorado y desarrollo del currículum*
}

Antonio Bolívar Botía ${ }^{1}$

\section{Resumen}

El artículo formula un análisis crítico de la formación permanente del profesorado, abogando por una formación centrada en el contexto de trabajo. En lugar de subordinar la formación docente a las necesidades de cambios impuestos por las políticas reformistas, se argumenta que la formación docente debe formar parte del propio programa de cambio e inscribirse en la trayectoria personal y en el establecimiento educativo en colaboración con los colegas. El desarrollo profesional, como comunidad de aprendizaje, debe vincularse al desarrollo personal y organizacional.

Palabras clave: Formación permanente de docentes, Desarrollo profesional, Innovación basada en la escuela, Comunidad profesional

\section{Summary}

The article formulates a critical analysis of the continuing professional training of the teachers, pleading for a professional development centred on the context of work. Instead of subordinating the teachers' in-service training to the needs of changes imposed by the political reformists, there is argued that the professional development effective must form a part of the own program of change and set within the personal and professional career, through collegial collaborative work in the same school. The professional development, as professional learning community, must link to the personal and organisational development.

Key words: Teachers' in-service training, Professional development, School-based improvement, Professional community

Continúa siendo una verdad incontrovertible, como en su momento aseveró Fullan (2002), que "la formación del profesorado sigue teniendo el honor de ser al mismo tiempo el peor problema y la mejor solución de la educación" (122). Esta paradoja da lugar a que, por un lado, haya proliferado un exceso de discursos sobre distintos modelos formativos, no siempre debidamente contextualizados y, al tiempo, pervivan en la práctica los formatos más tradicionales. En coherencia con las tesis mantenidas en otros trabajos (Bolívar y otros, 1999a), apostamos por una formación que articule las necesidades de desarrollo individual y las de la escuela como organización, donde los espacios y tiempos de formación estén ligados con los espacios y tiempos de trabajo, en que los lugares de acción puedan ser -a la vez- lugares de aprendizaje.

En este trabajo nos referimos preferentemente a la formación permanente de docentes, entendida como desarrollo profesional continuo del profesorado. Éste comprende todo el conjunto de actividades a las que los docentes se dedican a lo largo de su carrera, diseñadas para incrementar su competencia en el oficio. Como tal, es un proceso de

\footnotetext{
* Texto recibido el 5 de julio y arbitrado el 13 de agosto de 2004.

1 Catedrático de Didáctica y Organización Escolar en la Facultad de Ciencias de la Educación de la Universidad de Granada (España). E-mail: abolivar@ugr.es
} 
aprendizaje resultante de las interacciones significativas que tiene en el contexto temporal y espacial de su trabajo, y que da lugar a cambios en la práctica docente y en los modos de pensar dicha práctica (Kelchtermans, 2004). En sus formas más tradicionales se limita a cursos escolarizados de formación, mientras nuevas perspectivas han abogado por el aprendizaje con los colegas en el contexto de trabajo, vinculando el desarrollo profesional con el organizativo.

Por eso, a la hora de diseñar el desarrollo de un currículum, la formación del profesorado (inicial y permanente) desempeña un papel de primer orden, por lo que debe formar parte de su planificación y no quedar como una acción a posteriori, para aplicar los currículos previamente diseñados. En nuestro manual sobre Diseño, desarrollo e innovación del currículum, la formación del profesorado es un componente del diseño y desarrollo curricular. En su presentación del tema, Juan Manuel Escudero especificaba que "la formación y el desarrollo del profesorado se sitúan en el contexto de la diseminación de cambios, pero también en los propios de su desarrollo y evaluación, e incluso diseño o planificación... Ha de entenderse, por tanto, como un fenómeno transversal al currículum como un todo" (1999: 210).

Caben, además, otras argumentaciones complementarias. Un diseño y desarrollo curricular deben arbitrar los recursos necesarios para una adecuada puesta en práctica. Pero, en primer lugar, la relación entre recursos y buena implementación no es directa, sino dependiente del uso que se haga, principalmente por el profesorado ( $\mathrm{y}$, por tanto, de su capacitación). En segundo lugar, de hecho, como argumentan Cohen, Raudenbush y Ball, el capital social del profesorado (conocimiento, habilidades, pericia docente, etc.) con que contamos puede ser visto como uno de los recursos principales para que el desarrollo curricular tenga el impacto deseado en el aprendizaje de los alumnos. De ahí, creemos que una política coherente de reforma (Cohen y Hill, 2004) tiene que planificar la formación del profesorado dentro del propio proceso de diseño curricular. Es más, la propia formación del profesorado deberá estar basada en un visión curricular, y no en un catálogo de actividades dispersas, para escoger según preferencias. Como, entre otros, ha sostenido Susan Loucks-Horsley, el desarrollo profesional y el cambio educativo deben ir de la mano, pues la intención del primero es crear las condiciones para que el cambio pueda ocurrir en la acción.

Habitualmente, sin embargo, en la planificación de las reformas, el planteamiento ha sido distinto: hacer un diseño curricular $y$, posteriormente, recurrir a la formación del profesorado como un medio instrumental para poner en práctica los currículos diseñados previamente. Pero, cuando la formación del profesorado se subordina a programas externos de cambio, sin formar parte del propio programa, suele quedar como algo circunstancial, con una lógica adaptativa a posteriori a dichos cambios externos, en muchos casos, para persuadir de la bondad de la reforma, sin insertarse en la trayectoria de vida individual ni en un proyecto amplio de cambio educativo en el centro escolar. Para que la lógica de la reforma pueda transformarse en innovación, la formación del profesorado no puede concebirse sólo como un recurso instrumental para aplicar las propuestas externas; como parte del propio proceso de desarrollo, por el contrario, debe alimentar las dinámicas de cambio. Además, si queremos una mejora del currículum ofrecido, esto presupone un desarrollo profesional de los docentes, que no se producirá si no se reestructuran las instituciones educativas, de modo que no sólo proporcionen un aprendizaje de los alumnos, sino -en primer lugar- de sus propios profesores en dicho contexto de trabajo. 
Hablar, ante cualquier dificultad en la puesta en práctica de los nuevos currículos, de que el problema es debido a la falta de formación del profesorado suele ser una excusa para no entrar en cómo deban estar organizados los centros escolares y el ejercicio de la profesión docente. Se transfiere a otro lugar lo que es parte del propio problema. La formación del profesorado, en este sentido, llega a desempeñar la función de desviar ocultando sus límites- dónde está la verdadera cuestión. Además, silencia que el problema creado puede estar en el propio diseño externo, en su desconexión con la cultura profesional y escolar, o en la falta de medidas organizativas o políticas adecuadas como para que la formación sea una exigencia -no un prerrequisito- de la propia dinámica de cambio. Como señalaba Sarason en un libro con el deprimente título de El predecible fracaso de la reforma educativa,

El cambio no se producirá a menos que se cuestione la presunción de que las escuelas existen esencialmente para el crecimiento y el desarrollo de los niños. Esta presunción no es válida porque los maestros no pueden crear y sostener las condiciones para el desarrollo productivo de los niños si estas condiciones no existen para los maestros. (...) Los profesores han llegado a darse cuenta de que si no se consiguen las condiciones para su propio crecimiento, entonces no pueden crearlas y mantenerlas para los estudiantes (29 y 139).

En efecto, para la mejora escolar no bastan los cambios parciales en el currículum o en elementos aislados de la estructura de los establecimientos educativos, sin afectar al propio desarrollo profesional de los docentes. Sin una seria apuesta por reestructurar los centros y por rediseñar la profesión docente en la estructura organizativa de los centros, la añorada mejora educativa no ocurrirá, provocando -si acaso- la aversión del profesorado. $Y$ es que, finalmente, la innovación educativa no puede consistir en cómo implementar mejor sobre el papel buenos diseños externos, para pasar -más básicamente - a ser un nuevo modo de ejercer el oficio/profesión de enseñar y de funcionar las propias escuelas, como organizaciones y lugares de trabajo.

\section{Formación para aplicar los cambios}

Un enfoque tradicional, aunque habitual, centra su preocupación en el modo como los currículos diseñados externamente puedan ser implementados fielmente en las aulas, donde la formación continua del profesorado se convierte en un recurso ad hoc para dicha puesta en práctica. Este carácter instrumental también se apoya en el supuesto de que la función técnica de los profesores es aplicar los cambios decretados externamente y que habiendo sido diseñados por expertos, que les prestan la legitimidad requerida- su reproducción fiel será garantía de una mejora. En otros casos, las acciones específicas de formación -que se ofrecen en un amplio menú de cursos a la carta, para consumir por los clientes-, han funcionado con una estrategia consumista: incentivar el consumo otorgando créditos (por ejemplo, en España y Portugal) necesarios para la consolidación de subidas salariales. La formación del profesorado ha tenido así un valor de cambio, credencialista, no de uso de esa formación en el ejercicio profesional. Una formación, así entendida, se muestra poco preocupada por el desarrollo personal y profesional de los profesores, puesto que primariamente le importa la adaptación de los docentes a las nuevas exigencias del puesto de trabajo.

De acuerdo con el modelo dominante, se ha entendido que, para que un nuevo currículum funcione, es preciso suplir déficit en los modos tradicionales del saber hacer de los profesores, aportando los nuevos conocimientos/habilidades requeridos mediante los 
correspondientes cursos de formación. Este modelo de déficit asume que: a) la formación continua del profesorado puede ser concebida como un proceso definido y determinado externamente al centro y al profesorado, y b) que hay un cuerpo de conocimiento generalizable, universal y validado externamente que todo profesor puede aplicar en todo centro con el que llevar a cabo la innovación, su implementación y la consiguiente mejora de la práctica. Por eso, han predominado, en la práctica, formatos de formación continua del profesorado como una formación inicial retardada; por ello es preciso actualizar con nuevos conocimientos que suplan carencias iniciales, al margen de las necesidades individuales de profesionales adultos y de los contextos organizativos donde trabajan.

Formarse primero para aplicar los currículos después, en espacios y tiempos separados, es la materialización de una epistemología de la práctica, como aplicación técnica de la teoría, que criticara bien Donald Schön. La formación se convierte en un deus ex machina (el pilar fundamental de la reforma, creen sus gestores), que venga a resolver todos los problemas, ocultando sus limitaciones internas. Las estrategias y los formatos de formación se ponen instrumentalmente al servicio de la implementación de los cambios externos, con lo que implícitamente $-y$, sin duda, así ha sido percibido por los docentesse trata de adaptar a los profesionales a los cambios ya previamente diseñados, desligando formación y acción. Es un profesionalismo gestionado al servicio de la lógica del cambio institucional, al margen de la identidad profesional (Day y Sachs, 2004).

En lugar de entender la formación del profesorado como un recurso instrumental ad hoc para la adecuada puesta en práctica de una innovación curricular, con la consiguiente percepción de desprofesionalización y alienación, cabe entender el desarrollo profesional como una innovación en sí que, superando un enfoque individualista de la formación, se articule con el desarrollo organizativo del lugar de trabajo. La escuela debe ser no sólo el lugar de aprendizaje para los alumnos, como parece obvio, sino simultáneamente reestructurada de modo que sea un contexto estimulador del aprendizaje y crecimiento profesional de sus profesores y profesoras, donde la mejora en el conocimiento y la acción de los procesos de enseñanza-aprendizaje va en paralelo con los cambios en los procesos de trabajar en la escuela. La reclusión en el aula individual no llevaría muy lejos la innovación si, al tiempo, no se incrementan los modos de trabajar y aprender juntos (Joyce, 1990).

Los programas de desarrollo profesional, desconectados unos de otros, en los que se ofrece un conjunto de cursos a la carta (ofrecer diversos productos, como en cafetería, a elección), tienen escasa incidencia en el establecimiento escolar. Desde un enfoque más actual y comprensivo, al tiempo que se toma la innovación curricular como algo por generar desde el propio establecimiento, se integran los propósitos y las metas del profesor como persona (con sus vivencias, preocupaciones y ciclo de vida), el contexto real en que trabajan los profesores y la cultura de la enseñanza (Bolívar y otros, 1999a).

Este modelo escolarizado en la formación continua de adultos, realizado de manera concreta, como complemento o reciclaje de la formación inicial y poco articulado con las situaciones de trabajo, ha tenido escasas transferencias y efectos muy reducidos sobre las escuelas y en la actividad docente en el aula. La reproducción de este modo escolar de formación por parte de las ofertas institucionales ha llegado a tales límites de saturación en algunos países, que -tal vez- haya llegado el momento de repensar qué pueda ser la formación del profesorado, para incidir en otras propuestas alternativas (el lugar del trabajo como contexto formativo), que puedan contribuir a insertar la formación en las propias trayectorias de vida y proyectos de escuela. 
Estas críticas a la formación institucional externa no quieren ser una descualificación global ni una denostación fácil, para limitarse al conocimiento y a la práctica que en el propio contexto puedan tener los profesores. Sin duda el conocimiento profesional práctico necesita siempre ser revitalizado por su contraste con otras propuestas y enriquecido con las aportaciones del conocimiento pedagógico valioso disponible, en tanto que estructura normativa de la práctica (Eraut, 1994). Estimamos, por el contrario, que no todo -ni lo más relevante- puede aprenderse a través de ella. Deben coexistir ( $\mathrm{y}$, lo que es más importante, articularse) la formación instituida y la formación instituyente por la experiencia.

De hecho, un proceso de formación/innovación centrada en la escuela no excluye situaciones formalizadas de formación por parte de diversos especialistas externos (Domingo, 2001), pero sí las incluye dentro del proceso de trabajo y con un sentido particular (punto de llegada, más que de partida). En un clima inductor de dinámicas reflexivas e investigadoras, apoyado por la labor de asesoramiento, en determinados momentos del proceso, se puede plantear la necesidad, surgida desde las demandas de la práctica, de nuevos contenidos disciplinares, procesos o procedimientos para trabajar en el aula. Pero, en estos casos, se plantea siempre como una solución a problemas vividos, justo para hacer avanzar el desarrollo de la innovación.

\section{La formación como desarrollo profesional y organizacional}

Las modalidades de formación continua tendrán que insertarse en los propios establecimientos de enseñanza, como procesos de identificación de problemas, construcción de soluciones y planificación de proyectos de acción. Para que esto suceda, además de otras condiciones, se requiere una nueva relación entre poder y saber o, en otros términos, una gestión participativa y colegiada de los establecimientos educativos, que posibilite el liderazgo múltiple de los profesores, y donde una autonomía en el desarrollo curricular incite al desarrollo organizativo y profesional conjunto de colegios y escuelas.

Por eso, frente a la orientación instrumental de la formación permanente, abogamos -en un enfoque más constructivo- por la reapropiación de la experiencia adquirida para articularla con las situaciones nuevas de trabajo, en lugar de establecer una ruptura. Como enfoque constructivo, parte de que los profesores, como sujetos adultos (Mezirow, 1991), disponen de un conjunto de estructuras cognitivas, experiencias de vida y activos profesionales, etc., que deben ser punto de partida para la posterior reconstrucción de sus prácticas. En lugar de relegar, cuando no silenciar, los saberes profesionales y las experiencias de vida que han ido adquiriendo a lo largo de su carrera, se trata justamente de partir de ellas para contribuir al proceso de desarrollo personal y profesional. Aplicar un enfoque constructivista significa que el conocimiento se configura y reformula sobre la base de su interacción y transacción entre los significados previos y las nuevas experiencias en los contextos de trabajo. Esta construcción, además de individual, está situada socialmente en su entorno inmediato, que va cambiando (es decir, siendo reinterpretado) como resultado del propio proceso de aprendizaje, al procesar socialmente la información entre colegas. De ahí la relevancia de contextos que favorezcan el trabajo conjunto, la difusión de información, el intercambio de experiencias. El propio contexto organizativo se convierte en el lugar del aprendizaje y la reflexión sobre la práctica y, como tal, en la principal avenida de mejora (Santos Guerra, 2001). 
Se reclaman, pues, unos procesos formativos internamente generados, atentos a las situaciones y pertinentes a los contextos de trabajo, en los que los espacios y tiempos de formación -por una parte- y los espacios y tiempos de acción -por otra- no estén formalmente separados, si se apuesta por una autonomía creciente de los establecimientos educativos. El profesor adulto no es un objeto por transformar, para que deba ser sometido a un proceso formativo en los nuevos saberes, con la percepción desprofesionalizadora y alienante consecuente. Operando de este modo, se desdeña el amplio corpus de conocimiento y experiencias adquiridos en el ejercicio de una vida profesional, que ha configurado su propia identidad personal, con los prejuicios y creencias implícitas propias de una cultura profesional heredada, pero que son la base fundamental para reconstruir la práctica en función de una mejora.

No obstante, como -entre otros- ha resaltado Fullan (1992), el desarrollo profesional debe articular los propósitos y metas del profesor como persona y su integración en el contexto del colegio en donde trabaja. De este modo, resulta que generar cambios sustantivos en la enseñanza debe implicar repensar e integrar de manera radical el nivel individual/personal y el de la escuela como contexto de trabajo. Por eso, las estrategias formativas deben ser susceptibles de integrar, en un mismo proceso, cambios organizativos en las escuelas e individuales de los profesores, de modo que permitan la emergencia y la construcción de nuevas identidades y culturas profesionales. Pensar de un modo más holístico el desarrollo personal y profesional de los profesores y profesoras como individuos significa que los programas de desarrollo profesional no pueden limitarse a proporcionar determinados recursos o incentivos para cambios particulares, deben integrarse en una visión más amplia de la vida profesional de los profesores y las profesoras. Como afirma Fullan (1992), "el desarrollo profesional desde este punto de vista ha llegado a ser la suma total del conjunto de experiencias formales e informales acumuladas a lo largo de una carrera".

Los análisis biográficos, de carrera e identidad profesional o ciclos de vida, han puesto de manifiesto que no es posible disociar el desarrollo profesional y personal, por lo que es preciso articular los procesos formativos desde el punto de vista del que se forma, insertos en su trayectoria personal y profesional, de modo que pueda darse una implicación de las personas en el proceso formativo, en lugar de estar confeccionada de antemano desde la óptica de los agentes o instituciones externas de formación (Bolívar y otros, 1999a). El proceso formativo adquiere así los contornos de un proceso de desarrollo personal, de construcción de la persona del profesor, como reapropiación crítica -no de ruptura- de sus experiencias anteriores y modos de hacer, según criterios de pertinencia con las trayectorias profesionales. Esto no debe impedir lograr su congruencia con los intereses sociales y políticos más generales que, como servicio público, es la educación.

La formación permanente de docentes es, entonces, un caso particular de la formación de adultos. Como tal, puede aprovecharse todo lo que se ha aprendido en este ámbito, más específicamente en formación profesional de adultos, por lo que no debe limitarse a unas estrategias escolarizadas. Si el aprendizaje adulto consiste, entre otras cosas, en dar un nuevo significado a las acciones, por una transformación de perspectiva, será preciso partir de la experiencia profesional de vida, para que adquiera, así, nuevos significados o confirme los existentes, situándolo como sujeto y autor de su propia formación. La formación en personas adultas es primariamente una movilización de experiencias adquiridas, cuya reutilización con nuevos significados genera nuevos saberes. 
La reflexión colegiada sobre su propia praxis (metapraxis) y conocimiento propio (metacognición y autoconocimiento) es uno de los medios privilegiados del proceso de aprendizaje adulto. Como un profesional en desarrollo, la formación ha de venir dada por procesos de reflexión sobre la acción, con un carácter activo y en colaboración con colegas. Se trata de desarrollar un conocimiento profesional que se oriente a dar sentido a lo que hace, con una capacidad flexible de actuación, contextualizada, que se dirija preferentemente al uso y la aplicación. Partir, entonces, de un reconocimiento de experiencias, saberes y competencias adquiridos para ver en qué medida pudieran ser potenciados. Como adultos, son las situaciones de trabajo los contextos adecuados de formación para que la relación entre teoría y práctica sea fructífera. Como sensatamente dicen Hargreaves, Earl y Ryan,

Sería injusto, poco realista e inútil esperar que los profesores cambien espectacularmente su forma de enseñar, y que lo hagan, además, en un corto espacio de tiempo. Pero lo que sí es justo, realista y, probablemente, demostrará ser más efectivo, es esperar que se comprometan a procurar una continua mejora en su comunidad de compañeros, y que sean capaces de experimentar con las nuevas estrategias de enseñanza como parte de ese compromiso. Al tratar de mejorar las estrategias de enseñanza, a menudo nos inclinamos de manera entusiasta hacia la conversión, cuando hacerlo hacia la extensión sería un objetivo mucho más práctico y productivo (1998: 250).

Si la formación tradicional continua, en sus diversas modalidades, se ha mostrado -es una crítica común y compartida- inadecuada para resolver los problemas de la clase/establecimiento escolar, contamos con un amplio saber acumulado. A título de ejemplo reciente, una investigación sobre las formas más eficaces de aprendizaje del profesorado (Garet y otros, 2001), basada en una amplia muestra de profesores de secundaria, destaca seis aspectos:

- $\quad$ Forma. Redes de profesores o trabajo en grupo son más efectivas que las clases tradicionales y las conferencias.

- Duración. Programas sostenidos e intensivos tienen mucho mayor impacto que aquellos limitados y cortos.

- $\quad$ Participación colectiva. Son mejores las actividades diseñadas para profesores que trabajan juntos en el mismo centro, grado o disciplina.

- Contenido. Es clave focalizarlas tanto en lo que se enseña como en el modo de enseñarlo.

- $\quad$ Aprendizaje activo. Son más relevantes estrategias como observar y ser observada la enseñanza, planificación y puesta en práctica del aula, etc.

- $\quad$ Coherencia. Los profesores deben percibir el desarrollo profesional como parte coherente de otros programas de aprendizaje y enseñanza que se reflejan en sus centros, tales como la adopción de nuevos currículos.

En lugar de acciones puntuales sin conexión directa con las situaciones de trabajo, el proceso formativo debe movilizar los saberes poseídos en los contextos de trabajo a los momentos formativos, de modo que permitan reapropiar críticamente y recursivamente éstos a las nuevas situaciones de trabajo. Una relación de adecuación entre formación y trabajo puede provocar su reutilización, en las nuevas situaciones, de los saberes adquiridos anteriormente. Si bien se ha solido reconocer que los alumnos aprenden experiencialmente, construyendo a partir de lo que saben, hemos -por el contrariosupuesto que el problema de los profesores es que carecen de conocimiento, habilidades o destrezas, que deben ser remediadas por cursos de expertos, descontextualizados de su experiencia, para cada profesor como individualidad. 


\section{Aprender en el centro, construir el currículum}

Una de las cuestiones preocupantes para la mejora interna de la educación, como plantea Sarason en el texto citado anteriormente, es cómo rediseñar los establecimientos escolares para que no sólo sean un lugar de aprendizaje y trabajo para los alumnos, sino también, en primer lugar, para sus profesores y profesoras. Dado que no cabe esperar una mejora por prescripciones externas ni tampoco por buenas nuevas curriculares, parecería que sólo cuando el establecimiento educativo se convierta, como unidad básica del cambio, en constructor de la innovación, ésta repercutirá tanto en el aprendizaje y en la educación de los alumnos, misión última del sistema educativo, como en los agentes provocadores de dicho cambio: el propio profesorado. Como observa Elmore,

mejorar consiste en hacer lo correcto teniendo en cuenta el lugar donde se trabaja y no en el que se sabe al empezar a hacer el trabajo. La mejora es, en gran medida, una propiedad de las organizaciones, no de los rasgos preexistentes de los individuos que trabajan en ellas (2000: 25).

La formación permanente del profesorado no puede estar desconectada de los contextos de trabajo, como profesionales adultos debe articularse con ellos. La cuestión es, como plantea una buena experta en el tema (Little, 1999), cómo organizar los centros para el aprendizaje del profesorado. A este respecto señala las siguientes líneas productivas de acción:

- Poner en el núcleo del desarrollo profesional indagaciones colectivas sobre el aprendizaje de los alumnos. Todos los profesores aprenden de la experiencia por observación de sus alumnos, pero este aprendizaje informal e individual debe llegar a ser colectivo, sistemático y sostenido en el tiempo. Cuando el equipo de profesores se compromete en un diálogo o debate sobre la calidad del trabajo con los alumnos, o cuando analizan conjuntamente los resultados de la evaluación, esto tiene una influencia directa en el proceso de aprendizaje de los profesores.

- Organizar el trabajo diario de modo que apoye el aprendizaje del profesorado. Las rutinas diarias pueden impedir o contribuir al aprendizaje del profesorado, por lo que es importante organizar el trabajo de modo que lo promueva. Así, se puede hacer de acuerdo con una burocracia racional o de manera que puedan intercambiar conocimiento y habilidades. Tiempos para el diálogo común, por ejemplo, suelen ser imprescindibles.

- Desarrollar enfoques alternativos al aprendizaje del profesorado. Sabemos que determinados formatos son inadecuados para el desarrollo profesional, pero se pueden ensayar otras formas (equipos de investigación, reuniones, planificación común, observación en clase, etc.) que pueden promoverlo.

- Proveer feedback sobre el aprendizaje de los alumnos. Si los sistemas de evaluación externos, como rendimiento de cuentas, pueden dar datos, el uso que se haga de ellos depende del liderazgo local. Programas bien establecidos de revisión y evaluación del profesorado, unidos al aprendizaje de los alumnos, pueden enfocar el desarrollo profesional a sus metas clave.

- Desarrollar un ethos que apoye el aprendizaje del profesorado. Si las estructuras organizativas son importantes, también lo son los valores, las creencias y normas que favorecen el aprendizaje del profesorado. Así, es preciso entender el centro como una organización que aprende o el equipo docente como una comunidad profesional.

La formación ha de ir dirigida a cómo mejorar lo que se hace, desde un análisis de la situación, en que los propios procesos de trabajo sean en sí mismos generadores de cambios. La escuela debe ser reestructurada, si se quiere constituir en un espacio de 
aprendizaje e investigación no sólo para los alumnos, sino para los propios profesores: compartir conocimientos, preocupaciones y experiencias, en un aprendizaje de la propia práctica, lo que no exime de un necesario asesoramiento o apoyo externo, como dinamizador del propio proceso.

En parte, la argumentación subyacente sería (Smylie, 1995): si queremos que las experiencias educativas ofrecidas por las escuelas mejoren, los profesores y profesoras deben enseñar de manera distinta. Pero, para que esto suceda de modo relevante, no bastará el aprendizaje de nuevos contenidos o técnicas instructivas, sino -como ha puesto de manifiesto la teoría de la innovación- sus creencias y concepciones de la práctica, sus teorías implícitas de la acción. En orden a que esto pueda tener lugar habrá que partir, por una parte, de una adecuada comprensión de cómo los profesores, como profesionales adultos, aprenden y cambian; de otra, en lugar de dirigir los esfuerzos sólo a cómo cambiar los modos de actuar en papeles ya dados dentro de una estructura organizativa, intentar generar roles y estructuras que promuevan las prácticas educativas que deseamos.

El desarrollo curricular basado en la escuela comporta una determinada concepción de los profesores como profesionales reflexivos que investigan y comparten conocimientos en sus contextos naturales de trabajo, y exige ir configurando el establecimiento escolar (con los recursos y apoyos necesarios) como comunidad de aprendizaje para los alumnos, los profesores y la propia escuela como institución. La innovación centrada en la escuela y la formación continua del profesorado son dos procesos educativos paralelos, no sólo por realizarse dentro del centro escolar, sino porque uno y otro se co-implican. Si un nuevo desarrollo curricular implica aprender nuevas ideas y formas de hacer, la formación es intrínseca al propio proceso de desarrollo curricular, y no un medio para poder aplicar innovaciones externas.

El establecimiento escolar no debiera ser un lugar para colonizar con propuestas novedosas, sino base de regeneración del cambio, con los apoyos y condiciones ecológicas favorecedores. Se habla, por esto, de eco-formación frente a la heteroformación. La formación continua se inserta en los centros escolares como lugares de trabajo, donde el conjunto de experiencias y competencias individuales de sus miembros son el principal recurso formativo. Se trata, posibilitando tiempos y espacios, de que los profesores intercambien dichos conocimientos al servicio de la organización. La propia historia de la organización se constituye en un recurso de la formación. La formación centrada en la escuela supone también una reconceptualización del tiempo de formación: volver a dar a todo el tiempo su valor formativo, frente a recluir la formación al tiempo cautivo dedicado institucionalmente en un curso reglado a ella.

Ahora bien, no toda experiencia de trabajo provoca por sí misma un aprendizaje; para que no quede en mera impregnación o repetición rutinaria, se precisa un compromiso por aprender de las situaciones de trabajo. Cómo los establecimientos escolares puedan ser lugares de desarrollo profesional y aprendizaje de los profesores implica entrar en aquellas condiciones organizativas que restringen tal desarrollo. Por el otro lado, optimizar el potencial formativo de las situaciones y los contextos de trabajo pasa por crear dispositivos y dinámicas formativos (reflexión, trabajo conjunto, colaboración) que posibiliten la transformación de esas experiencias en aprendizaje profesional: oportunidades para trabajar y aprender unos de otros (de ahí la importancia de la colaboración en el trabajo y aprendizaje en grupo), unas relaciones igualitarias en el poder y autoridad en la toma de decisiones, así como la posibilidad de autonomía individual en el ejercicio del trabajo y de tareas. 
Parece también documentado en la literatura (De Vicente y otros, 2002), y evidenciado en las experiencias, que el intercambio de conocimientos y experiencias entre colegas suele ser uno de los medios más enriquecedores. Pero también que la cultura del individualismo y de la privacidad suele impedirlo. De ahí la llamada al trabajo en colaboración o equipo. Un incremento en la capacidad de toma de decisiones en cada escuela, en una vía de incrementar el compromiso en lugar del control, parece también ser una condición estructural en la estructura organizativa para implicar a los agentes y, de este modo, posibilitar trabajar juntos.

Y es que, al margen de la necesidad de determinadas acciones específicas, la formación permanente debe inscribirse en el contexto organizativo de los establecimientos educativos, de forma que permitan construir la innovación en el aprendizaje del centro escolar como institución. Por eso, resulta necesario estructurar estrategias de intervención formativa susceptibles de articular, en un mismo proceso, cambios organizativos en los establecimientos escolares y cambios individuales de los profesores, de manera que permitan la emergencia y la construcción de nuevas identidades y culturas profesionales. En suma se trata, en último extremo, de cambiar una cultura de la conformidad, de la dependencia y de la ejecución individual de propuestas externas, por una cultura de la autonomía, de la innovación internamente generada y del trabajo colegiado. La formación no es algo limitado exclusivamente a la persona del profesor individual, debiendo incluir dimensiones colegiadas, profesionales y organizativas.

Tomar en serio la idea de los establecimientos educativos como unidades básicas del cambio significa resituar la formación continua de los profesores de modo que, por una parte, contribuya a incrementar sus propios saberes y habilidades profesionales para reutilizarlos en las nuevas formas de hacer escuela; por otra, promover una formación centrada en la escuela, de modo que permita inscribirla en el propio proceso de construcción del cambio como tarea colegiada y en equipo. El problema de las reformas educativas deja de ser el problema de la formación, para trasladarse en cómo reestructurar los establecimientos escolares.

Si los profesores individuales pueden hacer poco cuando se enfrentan a las presiones, los límites de las prácticas colectivas y los hábitos institucionales establecidos, promover el cambio educativo como resolución de problemas significa ir construyendo comunidades profesionales de aprendizaje (Morrissey, 2000; Hord, 2003; Stoll, Fink y Earl, 2004), a través de la reflexión y revisión conjunta de la propia práctica, que incrementen su propia satisfacción y efectividad como profesionales en beneficio de los alumnos. Esta innovación organizativa es vista como un poderoso enfoque para el desarrollo profesional y una potente estrategia para el cambio y la mejora escolar. Si los beneficios son indudables y están documentados por la literatura, también los problemas para establecerlos son paralelos, pues suponen un cambio organizativo e individual de lo que se entiende por el ejercicio profesional. Las comunidades profesionales de aprendizaje suponen un paso más, con los apoyos que ha recibido de las organizaciones que aprenden (Bolívar, 2000; Senge, 2002), de las llamadas antes culturas de colaboración. Además, supera lo que ha sido una formación sectorial externa, limitada en el tiempo.

\section{El desarrollo profesional en la mejora de la enseñanza}

La formación del profesorado, en coherencia con otras líneas de actuación de asesoramiento y con las restantes estrategias de innovación, como metodología de trabajo de una formación/innovación centrada en la escuela, normalmente, utiliza un 
modelo abierto de resolución de problemas, con apoyo de asesores o agentes externos de cambio. La autorrevisión escolar, como reflexión conjunta de los profesores sobre sus prácticas, se puede constituir así en proceso y plataforma para el desarrollo y mejora interna de la escuela, y en la que habría de quedar integrada la formación continuada de sus profesores (Bolívar, 1999b).

El movimiento de investigación-acción ha contribuido a vincular el desarrollo curricular y el profesional, primero en una versión más individualista, y después, apostando por el trabajo en colaboración del establecimiento escolar como unidad de investigación y cambio. Como forma alternativa a un desarrollo curricular limitado a la implementación en las escuelas de un conocimiento externo, se plantea -como indagación autoreflexivareconstruir el desarrollo curricular y profesional como un proceso de investigación y cambio interno a la propia enseñanza. Los profesores son creadores e intérpretes del currículum, no simples implementadores. Para reconstruir el currículum lo adaptan y ajustan, basados en la observación y reflexión cooperativa sobre la experiencia, y contrastando con otros los conocimientos.

La práctica docente es vista como una actividad recursiva, de observar, cuestionar, alterar y evaluar los efectos de las prácticas intentadas. Se examinan críticamente los modos habituales de llevar el trabajo y los presupuestos que subyacen, para tener comprensiones nuevas de sí mismo, de las situaciones de enseñanza y de las propias asunciones implícitas en estos contextos y modos de hacer. El análisis de la práctica, en un ciclo reflexivo colegiado, genera -en diferentes niveles- nuevas comprensiones de ella. Como resultado de confrontar los problemas genera -en diferentes niveles- nuevas comprensiones de ella. Como resultado de confrontar los problemas, y las soluciones dadas hasta ahora, los modos de ver la enseñanza se recomponen de manera diferente, lo cual puede provocar acciones distintas (Pollard, 2003).

A partir de la autorrevisión conjunta de la práctica docente, en la que se realiza un diagnóstico de la situación, examinando los modos habituales de pensar y hacer, se consolida -como logros- lo que se está haciendo bien (¿De qué aspectos/elementos de $\mathrm{mi} /$ nuestra enseñanza nos podemos sentir relativamente contentos?), al tiempo que se reconoce e identifica lo que deba ser mejorado (¿En qué aspectos debo/debemos incidir o cambiar para mejorar nuestra enseñanza-aprendizaje?). Tras llegar a compartir una percepción común de los problemas o necesidades, se debe entrar en la fase de movilizar las experiencias, los saberes y las ideas para proponer los cambios deseables y encontrar las soluciones posibles en las pautas de trabajo en clase/centro. Junto al espacio del aula se analizan aquellos factores del sistema escolar o del centro que condicionan la propia enseñanza (¿Qué factores de la organización del centro deben cambiar para posibilitar lo anterior?, o ¿a qué intereses, y a cuáles no, está sirviendo este modo de hacer las cosas?).

El propio proceso de enseñanza debe ser concebido como un proceso de investigación, formativo para los propios profesores, en un contexto institucional de las escuelas que lo favorezca, que -sin excluir el apoyo de agentes externos- rompa con la tradicional hegemonía del conocimiento externo académico. Las comunidadesde- práctica (Wenger) son personas comprometidas en resolver problemas, con las cuales se comparte el conocimiento tácito y se saca a la superficie por el diálogo, se intercambian ideas sobre el trabajo y se experimenta con nuevos métodos o ideas, lo que motiva a modificar las teorías-en-uso. El crecimiento, como profesional del profesorado, se inscribe en el desarrollo del establecimiento escolar donde trabaja; de allí que si queremos nuevas 
prácticas docentes y patrones de relaciones entre los profesores, esto conduce paralelamente a la reestructuración de los contextos organizativos en que trabajan.

El currículum, como misión y oferta socio-educativa de la escuela, es algo por construir en un proceso de deliberación y decisión comunitarias. A su vez, el desarrollo curricular no estaría separado del diseño y de la planificación (unos diseñan y otros, trabajadores, realizan); por el contrario, estaría integrado y al servicio del crecimiento del propio establecimiento como organización y de los profesores como profesionales. La innovación/formación como mejora versa sobre algunos ámbitos curriculares específicos, que se toman como prioritarios y sobre los que se decide introducir cambios. Aun cuando la práctica educativa tiene un carácter personal, los criterios de actuación no quedan al arbitrio de cada profesor; son, por el contrario, un tema de deliberación y construcción por parte de la escuela en su conjunto. La innovación curricular se concibe como un proceso cíclico de experimentación, creación, investigación, y evaluación de su práctica en sus centros.

El proceso de innovación y formación, en paralelo a los ya señalados, normalmente surge por la identificación, en un ciclo de reflexión colegiada o diálogo entre compañeros, apoyados inicialmente por algún agente de cambio externo, de ámbitos o campos donde existen déficit o discrepancias entre lo que se hace y lo que se declara/espera hacer, porque no se han obtenido los resultados esperados/deseados o por haberse embarcado en un proceso de autorrevisión. De este modo, identificar situaciones deficitarias o insatisfactorias es, entonces, un punto necesario y originario para generar acciones para la mejora. Este proceso de autorrevisión se va constituyendo en un marco para discutir, deliberar y decidir colegiadamente qué conviene hacer, cómo van las cosas y qué habría que corregir. El proceso debe abocar a acuerdos prácticos (qué hacer y cómo hacerlo), siendo muy importante - para asegurar su posterior traducción práctica - el propio proceso de diálogo, confrontación de perspectivas y acuerdos finales logrados. El trabajo conjunto del grupo de profesores del departamento o colegio, en un proceso de discusión, deliberación y decisión del estado actual de su labor docente, es la plataforma para emprender acciones de formación y mejora. La autoevaluación de la situación por los docentes, una vez convertida en forma habitual de trabajo, compartiendo logros y necesidades, será, entonces, el contexto de la formación y de la innovación.

La formación y la innovación centradas en la escuela, en lugar de ser una estrategia instrumental y gerencialista para aceptar y poner en práctica reformas externas, puede considerarse una de las principales plataformas teóricas y prácticas para cambiar la cultura tradicional de los establecimientos escolares, para promover la profesionalidad de los profesores y la innovación. Por eso uno de los retos más importantes de la formación del profesorado, frente a las prácticas dominantes al uso, es incardinarla en sus contextos de trabajo para que sea significativa y relevante; contribuir a reconstruir la cultura profesional de colaboración pedagógica, promoviendo espacios de formación y formas de hacer conjuntos, y conectar la formación con el desarrollo y la mejora del currículum escolar, articulando la formación sobre el análisis colegiado de ámbitos de mejora en la escuela y los consiguientes proyectos de trabajo. En este camino, la formación continuada del profesorado en los establecimientos educativos, como estrategia de desarrollo curricular basado en la escuela, está llamada a desempeñar una alternativa a modos tradicionales de trabajar en educación.

Dentro del movimiento de enfocar la innovación en la mejora de los resultados de los alumnos y en la presión actual por el rendimiento de cuentas, también la formación del profesorado adquiere nuevas metas. Así, Cohen y Hill sostienen que las iniciativas 
políticas de cambio curricular, si quieren mejorar el aprendizaje, deben dirigirse a tres componentes clave del desarrollo profesional: conocer las metas y los propósitos del nuevo currículum, practicar las metodologías de enseñanza que posibilitan la consecución de las metas deseadas y explorar el tipo y contenido de evaluación que los alumnos deban afrontar. Una política educativa coherente o sistémica debe coordinar los cambios a diversos niveles: curricular, organizativo y profesional.

Si la mejora debe producirse a nivel de cada escuela, la mitad de esa mejora es la enseñanza del profesor en el aula, que no puede ser minusvalorada en función del establecimiento como conjunto. De ahí que las oportunidades para aprender de los profesores deben ser una prioridad en la planificación de los cambios curriculares, dada su influencia crucial en lo que los alumnos aprendan. En efecto, la mejora de la calidad de la enseñanza pasa por una formación del profesorado de mayor calidad y un desarrollo profesional más relevante. No obstante, recogiendo lo que hemos aprendido a nivel de centro, se trata de conjugar la formación personal con la creación de comunidades profesionales, en una organización colegiada de la enseñanza que posibilite construir la capacidad interna de mejora, con los oportunos apoyos externos.

Por eso, una comunidad profesional no pretende alcanzar mayores niveles de colaboración entre los profesores como un fin en sí mismo; es un medio para la finalidad básica de la institución escolar: el núcleo del trabajo conjunto debe ser el currículum escolar, con el objetivo prioritario de mejorar el aprendizaje de los alumnos. De este modo, las políticas de desarrollo profesional se deben dirigir a incrementar la capacidad de la escuela para mejorar, cuyo foco es la calidad de la enseñanza ofrecida, que se tendrá que reflejar en los niveles de consecución del alumnado. Resumiendo el saber adquirido, Fred Newman et al. (2000), dicen:

El desarrollo profesional de los profesores ha sido a menudo recomendado como una estrategia para la mejora de la escuela. Pero el desarrollo profesional, generalmente, ha fracasado a la hora de mejorar la enseñanza, porque normalmente ha sido implementado en modos que violan condiciones clave para el aprendizaje del profesor. Los investigadores están de acuerdo en que para promover un aprendizaje del profesorado que conduzca a la mejora de la enseñanza, el desarrollo profesional debe concentrarse en la enseñanza y resultados de los alumnos en cada centro, proveer oportunidades para la investigación colegiada, ayuda y feedback, y conectar el apoyo de asesores externos con el respeto de la discreción y creatividad individual de cada profesor. Las experiencias, finalmente, han de ser sostenidas y continuas, más que cortas y episódicas. (...) Además, el desarrollo profesional que más probablemente incrementa los niveles de consecución de todos los alumnos en una escuela es aquel que no sólo se dirige al aprendizaje individual de cada profesor, sino también a otras dimensiones de la capacidad organizativa de la escuela (259-260).

\section{Referencias bibliográficas}

Bolívar, A. Cómo mejorar los centros educativos. Madrid: Síntesis, 1999b.

Los centros educativos como organizaciones que aprenden. Promesa y realidades. Madrid: La Muralla, 2000.

Bolívar, A. (dir.) y otros (1999). Ciclos de vida profesional del profesorado de Secundaria. Desarrollo personal y formación. Bilbao: Mensajero, 1999a. Edición portuguesa: Profissão 
Professor: O itinerário profissional e a construção da escola. Bauru/São Paulo (Brasil). Editora da Universidade do Sagrado Coração, 2002.

Cohen, D. K. y Hill, H. C. Learning policy. When State education reform works. New Haven: Yale University Press, 2001. Cohen, D. K., Raudenbush, S. W. y Ball, D. L. "Resources, instruction, and research", en Mosteller, F. y Boruch, R. (eds.). Evidence matters: Randomized trials in education research. Washington, DC: Brookings Institution Press, 2002, pp. 89-119. Obtenido [22/10/02] en: http://wwwpersonal.umich.edu/ dkcohen/DKC_Recent_Pubs.html. Una versión anterior es obtenible también en: http://www.sii.soe.umich.edu/links.html

De Vicente, P. (dir.) y otros. Desarrollo profesional del docente en un modelo colaborativo de evaluación. Bilbao: ICE de Universidad de Deusto, 2002.

Day, C. y Sachs, J. (eds.). International Handbook on the Continuing Professional Development of Teachers. Berkshire: Open University Press, 2004.

Domingo Segovia, J. (coord.). Asesoramiento al centro educativo. Colaboración y cambio en la institución. Barcelona: Octaedro/Ediciones Universitarias de Barcelona, 2001.

Elmore, R. F. Building a new structure for school leadership. Washington, DC: Albert Shanker Institute, 2000. Trabajo obtenible en [consulta 5/09/02]: http://www.shankerinstitute.org/

Eraut, M. Developing professional knowledge and competence. Londres: RoutledgeFalmer, 1994.

Escudero, J. M. (ed.). "El currículum, las reformas y la formación del profesorado", en Escudero, J. M. (ed.), Diseño, desarrollo e innovación del currículum. Madrid: Síntesis, 1999, pp. 209-236.

Fullan, M. Succesful school improvement. The implementation perspective and beyond. Buckingham: Open University Press, 1992.

---- Las fuerzas del cambio. Explorando las profundidades de la reforma educativa. Madrid: Akal, 2002.

Garet, M., Porter, A. C., Desimone, L., Birman, B. F. y Yoon, K. S. "What makes professional development effective? Results from a national sample of teachers", en American Educational Research Journal, 38. Núm. 4 (2001): 915-945.

Hargreaves, A., Earl, L. y Ryan, J. Una educación para el cambio. Reinventar la educación de los adolescentes. Barcelona: Octaedro, 1998.

Hord, S. M. Learning Together, Leading Together: Changing Schools Through Professional Learning Communities. Nueva York: Teachers College Press, 2003.

Joyce, B. (dir.) Changing school culture through staff development. 1990 ASCD Yearbook. Alexandria, Va: Association for Supervision and Curriculum Development, 1990.

Kelchtermans, G. "Continuing Professional Development for Professional Renewal: Moving Beyond Knowledge for Practice", en C. Day y Sachs, J. (eds.). International 
Handbook on the Continuing Professional Development of Teachers. Berkshire: Open University Press, 2004.

Little, J. W. "Organizing schools for teacher learning”, en Darling-Hammond L. y Sykes, G. (eds.). Teaching as the learning profession: Handbook of teaching and policy. San Francisco: Jossey Bass, 1999, pp. 233-262.

Loucks-Horsley, S. "Managing change: an integrated part of staff development", en Staff Development: A Handbook of Effective Practices. Oxford, $\mathrm{OH}$ : National Staff Development Council, $1998 . \quad$ También on-line [25/05/04] en http://www.enc.org/professional/learn/change/resources/readings/

Mezirow, J. Transformative dimensions of adult learning. San Francisco: Jossey-Bass, 1991.

Morrissey, M. S. Professional Learning Communities: An ongoing exploration. Austin, Texas: Southwest Educational Development Laboratory, 2000. Disponible [23/03/04] en:

Newmann, F., King, M. B. y Youngs, P. "Professional development that addresses school capacity: Lessons from urban elementary schools", en American Journal of Education, vol. 108. Núm. 4 (2000): 259-299. Una versión de su presentación como paper en la AERA se puede obtener en línea [14/06/04]:

Pollard, A. (ed.) Reflective teaching: effective and evidence-informed professional practice. Londres: Continuum, 2003.

Santos Guerra, M. A.: Enseñar o el oficio de aprender: Organización escolar y desarrollo profesional. Rosario/Santa Fe: Homo Sapiens, 2001.

Sarason, S. B. El predecible fracaso de la reforma educativa. Barcelona: Octaedro, 2003.

Schön, D. La formación de profesionales reflexivos: hacia un nuevo diseño de la enseñanza y el aprendizaje en las profesiones. Barcelona: Paidós, 1992.

Senge, P. Escuelas que aprenden. Bogotá: Norma, 2002.

Smylie, M. A. "Teacher learning in the workplace: implications for school reform", en Guskey, T. R. y Huberman, M. (eds.). Professional development in education; new paradigms and practices. Nueva York: Teachers College Press, 1995, pp. 92-113.

Stoll, L., Fink, D. y Earl, L. Sobre el aprender y el tiempo que requiere: implicaciones para la escuela. Barcelona: Octaedro, 2004.

Wenger, E. Comunidades de práctica: aprendizaje, significado e identidad. Buenos Aires/ Barcelona: Paidós, 2001. 